MS40-04

\section{Crystallophore, a unique nucleating and phasing agent for biocrystallography}

Eric Girard ${ }^{1}$, Sylvain Engilberge ${ }^{1}$, Tristan Wagner ${ }^{2}$, François Riobé ${ }^{3}$, Olivier Maury ${ }^{3}$

1. Institut de Biologie Structurale, GRENOBLE, France

2. Max Planck Institute for Terrestrial Microbiology, MARBURG, Germany

3. École Normale Supérieure, LYON, France

email: eric.girard@ibs.fr

With $90 \%$ of the structures present in Protein Data Bank, crystallography is one of the methods of choice for obtaining structural information on proteins. However, the method still suffers from the two inherent locks: obtaining single crystals and solving the phase problem. The crystallization of proteins thus remains the major lock and we consider that approximately one purified protein out of three will lead to exploitable crystals and this, in spite of the exploration of hundreds of physico-chemical conditions allowed by the crystallization robots and the development of numerous crystallization kits. Another approach is the use of nucleating agents (heterogeneous nucleation) to lower the energy required to form the crystalline germ such as Molecular Imprinted Polymers (Saridakis \& Chayen, 2013).

We recently proposed a new molecule, called Crystallophore (Xo4), which acts as a nucleating agent (Engilberge et al., 2017). Xo4 is a lanthanide complex formed from a molecular cage chelating a lanthanide atom. We will present the results obtained on about fifteen proteins which show that Xo4 induces unique crystallization conditions (Figure 1a) and promotes new crystal packing thanks to supramolecular interactions.

The lanthanide atom present in Xo4 also makes it possible to obtain an important anomalous signal that greatly facilitates the phase determination process, as illustrated by various examples (Engilberge et al., 2017; Vögeli et al., 2018) (Figure 1b).

The versatility of the interactions between Xo4 and the surface of proteins explains its exceptional properties making this molecule a unique tool for simultaneously solving the two major locks of biocrystallography.

Figure 1: a) Examples of crystallization drops obtained without (top) and with Xo4 (bottom) for three different proteins. b) Structure of the complex thiolase/HMGCS/ DUF35 involved in the synthesis of mevalonate (Vögeli et al., 2018).

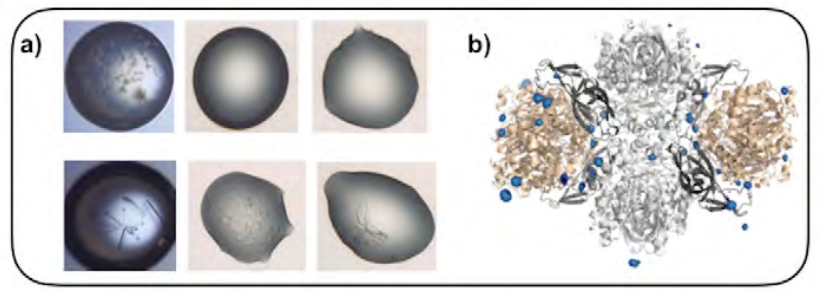

References:

Engilberge, S., Riobé, F., Di Pietro, S., Lassalle, L., Coquelle, N., Arnaud, C.-A., Pitrat, D., Mulatier, J.-C., Madern, D., Breyton, C., Maury, O. \& Girard, E. (2017). Chem. Sci. 9, 5909-5917.

Saridakis, E. \& Chayen, N. E. (2013). Trends Biotechnol. 31, $515-520$.

Vögeli, B., Engilberge, S., Girard, E., Riobé, F., Maury, O., Erb, T. J., Shima, S. \& Wagner, T. (2018). Proceedings of the National Academy of Sciences. 115, 3380-3385.

Keywords: Protein crystallization, structure determination, crystallophore 